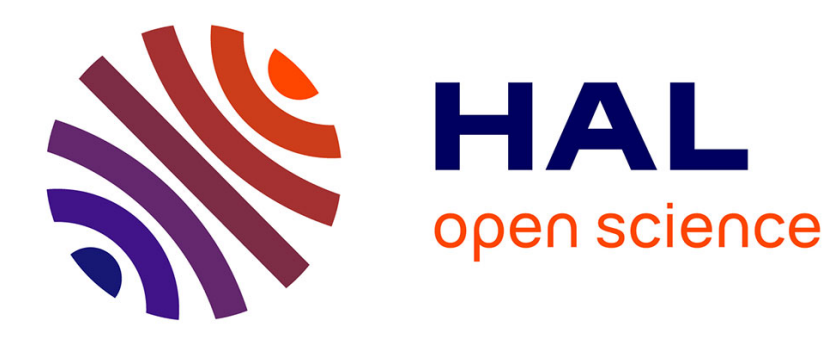

\title{
De l'anthropologie au développement et retour
}

J. Barette

\section{To cite this version:}

J. Barette. De l'anthropologie au développement et retour. Revue Tiers Monde, 2007, 191, pp.591-610. ird-00405436

\section{HAL Id: ird-00405436 \\ https://hal.ird.fr/ird-00405436}

Submitted on 20 Jul 2009

HAL is a multi-disciplinary open access archive for the deposit and dissemination of scientific research documents, whether they are published or not. The documents may come from teaching and research institutions in France or abroad, or from public or private research centers.
L'archive ouverte pluridisciplinaire HAL, est destinée au dépôt et à la diffusion de documents scientifiques de niveau recherche, publiés ou non, émanant des établissements d'enseignement et de recherche français ou étrangers, des laboratoires publics ou privés. 


\section{De l'anthropologie au développement et retour.}

Jean-François Baré $\left({ }^{1}\right)$

On pourrait sans doute commencer ainsi l'exercice un peu proustien auquel me convie cette revue : longtemps je me suis comporté comme un anthropologue classique. Le développement était un douteux fatras d'ethnocentrisme, de paysanneries déplacées et de cheminées d'usine, de généralisations ennuyeuses et de surcroît porteuses d'échecs dus à l'ignorance abyssale des fonctionnaires, technocrates et hommes politiques qui s’y employaient ; ils ne connaissaient rien aux « cultures locales » où aurait été répandue une sorte de sagesse immémoriale enfouie par le discours dominant de l’Occident sur le monde, dont nous avions à témoigner.

Je voudrais donc expliquer comment je me suis vite départi de cette position, mais aussi comment j'ai essayé de tourner un regard anthropologique vers ce domaine multiforme, pour trouver finalement que le monde du développement était un monde spécifique, peu traduisible entre ses acteurs, spécifiquement constitué, et qu’il ressemblait de manière frappante à un objet d'étude anthropologique; sans pour autant qu'il relève de la fiction, bien au contraire. Au cours d'une vie déjà un peu longue je m’aperçois avoir déjà publié -ou édité- une quantité assez massive de pages imprimées sur ce « sujet », et je prie le lecteur de m’excuser de l’y renvoyer un peu trop souvent sans doute, à seule fin de ne pas trop me répéter.

Je préfère dès à présent résumer ce qui va suivre en quelques phrases

- la notion de développement relève des catégories locales de Geertz ou des catégories de langue de Benveniste, elle est indissolublement liée à celle de croissance économique dans le

$\left({ }^{1}\right)$ Anthropologue, directeur de recherche à l’I.R.D. 
champ intellectuel de l'économie politique. Elle n’est pas à proprement parler définissable en elle-même (1987)

-ce qui serait l’anthropologie du développement n’est donc pas définissable non plus (1995) -une anthropologie appliquée au développement ne peut être qu’une réflexion très descriptive à destination des institutions publiques (para-publiques, privées) ayant pour vocation ce qui serait le développement dans quatre domaines au moins : l’incorporation du savoir anthropologique en amont de la décision et donc une ethnographie finalisée des institutions d'aide ; la description de faits de communication (ou de non communication) linguistique; l'évaluation des projets et politiques de développement, considérée comme le projet de « raconter une histoire vraie » (1995 c, 2001).

\section{L’anthropologie comme littérature réaliste}

Je croyais (et d'ailleurs je le crois toujours par certains côtés), que le monde humain, même avec sa violence et ses déchirements, avec sa convoitise et ses vertus, n’était qu’un immense paysage extraordinairement varié d’énoncés d'une faramineuse poésie, de cette poésie qui selon Eluard avait pour but « la vérité pratique ». Cette poiesis me paraissait réduire ce qui aurait été le corpus des théories du développement à une série de vagissements infantiles, aussi lourds et invraisemblables que répétitifs . C’était un monde où l'on disait « un parent par alliance est une hache d'éléphant », ou bien « le Verbe s’est fait chair et a habité parmi nous »; où la possédée d’une « reine » sakalava du Nord de Madagascar pouvait signifier que celle qui l’ »habitait » ne souhait pas être enterrée au tombeau royal de Tsinjoarivo parce que les dignitaires chargés du rituel avaient vu émerger en creusant la tombe des « pierres énormes » (vato maventy). Ceci avait provoqué un nouvel enterrement, qui avait à nouveau déplacé de véritables foules. Pour parler déjà en termes économiques, ce « conflit » avait certes provoqué des « effets induits », en termes d'achats de bœufs pour les sacrifices, de 
construction de pirogues et autres consommations d'un rhum produit par les périmètres de canne à sucre du Nord de Madagascar. En même temps cette proposition m'aurait paru assez comique et ce serait toujours le cas à présent. C’eut été un peu comme dire que le concile de Trente avait eu pour fonction d'enrichir les pâtissiers italiens. $\left({ }^{2}\right)$

Dans ce sens l'anthropologie, contrairement à l'économie et notamment à l'économie du développement, me paraissait ressortir d'une position éminemment réaliste, et je trouvais absolument merveilleux et pour tout dire surprenant qu’une entreprise dédiée à « rendre compte des sociétés dans leurs propres termes », pour citer l’expression de Georges Condominas, eut droit de cité à un tel point qu’elle était enseignée en Faculté, à Paris X Nanterre et ailleurs. L’anthropologie que j’aimais c'était celle de Condo, celle du Geertz de Bali et des « catégories locales » (1983) $\left({ }^{3}\right)$, celle de mon regretté ami Paul Ottino qui, voyant un dénommé Tihiva Autai, 70 ans, tirer d’une pirogue un masque et un fusil de pêche sous marine, répondait à l’interrogation muette en disant qu’il « n’était pas vieux, car étant seul il ne pouvait pas se permettre de l’être » (1972). Celle de Claude Lévi-Strauss aussi, pour la fascinante rigueur des Structures Elémentaires de la Parenté et au début de son œuvre celle de cette « logique du malheur » des sociétés de Basse Côte d’Ivoire restituée de si étincelante manière par Marc Augé. (1975)

L’anthropologie telle que la découvrais plus jeune et assez voracement me paraissait institutionnaliser la littérature réaliste, et permettre d’espérer pratiquer officiellement ce plaisir frauduleux que je trouvais tout jeune à l’Ulysse de James Joyce -une seule journée dans Dublin !- ou bien plus tard celui qui me ferait passer des heures comme sans y penser à

$\left(^{2}\right)$ Le faux dilemme entre raison culturelle et raison pratique a été remarquablement exploré par Marshall Sahins dans Culture and Practical Reason, Chicago University Press, 1976 ( très mauvaise traduction en 1980 dans la Bibliothèque des Sciences humaines sous le titre Au cour des sociétés, chez Gallimard.)

$\left({ }^{3}\right)$ Mais certainement pas celle du Geertz relativiste et « post-moderne ». 
la lecture de Jorge Amado. Je connaissais déjà un peu du Brésil par des livres de sociologie, et je trouvais dans Gabriela girofle et cannelle ou dans Bahia de tous les Saints une efficacité descriptive que l’ abondante et répétitive littérature sociologique -ou « socio-géographique », ou » géo sociologique ? » sur les « fronts pionniers » brésiliens ne me paraissait pas toujours égaler. La violence nue des commandants de plantation envers les petits et les faibles, (sans parler des Indiens dont on se demandait là comme ailleurs s’ils étaient vraiment humains, tels les pauvres eux-mêmes) y apparaissait comme la clé de voute de tout discours, à partir de laquelle seule l'amour, la séduction et la recherche lancinante de l’argent du lendemain pouvait s’inscrire dans le monde perçu.

\section{Le relativisme culturel, l’anthropologie et le développement}

La littérature anthropologique était-elle si réaliste que ça ? Certes je ne regrette pas d'avoir infligé aux lecteurs de Sable rouge par exemple une série assez répétitive de chroniques familiales de groupes roturiers du Nord-Ouest de Madagascar, pour cette bonne raison que pour ces paysans l’Histoire avec un grand H c’était la succession de leurs « grands », et aussi qu’on y voyait émerger des récurrences, l'émergence de groupes résidentiels dominants à l'intérieur de l'évolution de vastes groupes de descendance indifférenciés, des relations d'une inégale proximité avec ces centres de pouvoir locaux que constituaient les résidences royales et les tombeaux royaux. La chronique des segments royaux était ontologiquement moins répétitive, pour cette raison qu’à chaque succession c’était toute une saga qui était rejouée, dont témoignaient des noms posthumes (fitahiana) emportant à eux seuls une « histoire », presqu’au sens commun: ainsi Andriamamalikiarivo, « la souveraine qui a dispersé mille 
personnes », (Tsiomeko de son vivant) renvoyait à cette période charnière des années 1840 où des groupes islamisés montaient en puissance par opposition aux Sakalava « historiques ».

Sans doute peut-on comprendre, rétrospectivement, que je me sois laissé aspirer par ces constructions baroques et que je ne me sois assez peu soucié des conditions proprement économiques de la vie de ces gens. Je considérais que ce n’était pas mon problème et d'ailleurs je trouvais qu'il n'y avait pas grand-chose à en dire de très intéressant. On sait que depuis quelques décennies la notion de « pauvreté » a été placée sur le devant de la scène par de puissantes institutions multilatérales dont la Banque mondiale, ce qui peut faire sourire lorsque l’on se demande de quoi s’occupaient auparavant ces institutions là ; mais peut-être fallait-il, paradoxalement, que l'on prononçât le mot pour que le problème soit posé, comme dans les célèbres argumentaires de Victor Hugo.

Pourtant si les abandons d'enfant étaient inconnus des communautés rurales du Nord de Madagascar, les gens qui vivaient au seuil de la pauvreté ne manquaient pas.

Peut-être est ce genre de dureté qui m’a fait rechigner devant le projet de décrire les conditions concrètes de la vie : la dureté même des Sakalava faite aux femmes, la dureté des femmes envers leurs conjoints envers qui elles pratiquaient un chantage perpétuel à l'amour pour conserver quelques biens personnels, cette dureté là était trop évidente et contrariait le tableau que je me faisais de la solidarité de communautés rurales exotiques. Pourtant, pour le fanatique des « catégories locales » que j’étais, il n’en manquait point à cet égard. Ce que le gens faisaient d'abord de leur vie, me disait-on, c'était qu'ils mijaly, qu’ils « manquaient », ce qui voulait souvent dire manquer de viande. Les gens n’avaient pas vraiment de « règles » (didy) me disait -on parfois, ce qu'ils cherchaient c'était de fivelomantena, c'est-à-dire de « faire vivre leur personne », ou autrement dit de trouver « quelque chose à bouffer », jour après jour. 
S’immerger dans cette affaire dépassait mes compétences, et de toute évidence je crois qu’elle a dépassé les compétences de pas mal de gens pendant pas mal de temps. Je trouvais confusément que c’était une affaire d'ordre politique dont je n’avais pas à me mêler, mais je m’apercevrais plus tard que c’était une tautologie de Café du Commerce, qu’il fallait spécifier. Je ne trouvais rien, mais absolument rien, de compréhensible et encore moins d’utilisable hic et nunc, à Madagascar, dans l'épuisante logorrhée marxiste qui tenait lieu de critique sociale dans les années 1970, bien que j’avais lu Marx tout autant et parfois même plus que d'autres. Et je ne voyais pas de plus pour quelle raison j'aurais eu à me mêler de faire la révolution à Madagascar à la place des Malgaches, et selon quelles médiations. Des « intellectuels de gauche » français entourés d’épigones Malgaches à la fois fascinés et haineux, dénonciateurs d’un « néo colonialisme » dont ils ne s’apercevaient pas à quel point ils le pratiquaient sous des formes nouvelles, s’en chargeaient d’ailleurs, généralement dans un rayon de 100 kilomètres autour de Tananarive, ou peut-être l’ont-ils cru un moment.

D’ailleurs, on ne me trouvera peut-être pas trop paradoxal si je dis que le mot de « pauvreté » ne convenait pas tout à fait pour les paysans du Nord tropical de Madagascar. C'étaient certes des gens loqueteux, qui n'avaient pas beaucoup de chemises de rechange, et dont les maillots de corps étaient parfois en lambeaux ; c’étaient des gens si dignes qu’ils se rendaient les uns aux autres des boites de conserve usagées, qui servaient de pommes de douche, et qui s’étonnaient qu’on puisse jeter l'objet technique faramineux qu’était un briquet. Mais contrairement à leurs dires ils mangeaient en gros à leur faim, sacrifiaient des bœufs assez régulièrement pour de grands festins de viande, ils péchaient, ils riaient beaucoup (surtout de questions sexuelles, mais aussi du comportement des pintades qui formaient des personnages réguliers des contes du soir, de bien d'autres choses), ils dormaient en milieu de journée. Ils n’étaient pas trop « pauvres en temps » contrairement aux femmes du Sahel si justement 
évoquées par Jacques Charmes dans cette revue. Ce que j’aurais appelé la pauvreté c’était de revenir après huit heures à la chaîne et à la nuit tombée en banlieue parisienne, pour un « Smicard » dont le loyer devait déjà engloutir un bon quart du revenu, et dont les fins de mois n’étaient pas plus assurées que celles de Ainée de Husseini. Ou bien, toujours à Madagascar, celle des femmes Tandroy du Sud sub-désertique, qui avaient parfois à porter l'eau sur des kilomètres, et qui envoyaient leurs fils faire les tireurs de pousse dans le port de Tuléar, sous peine de survie. Le standard de « pauvreté absolue » de la Banque mondiale, récemment passé de 1 à 2 US dollars par jour, ne me parait décrire qu’ imparfaitement ce genre de contexte, sans doute parce que c’est un standard précisément, et que la mesure monétaire ne dit pas tout.

Ce qui m’a poussé à m’intéresser aux questions de développement, c'était tout autant l'attention perplexe que j'ai portée aux conditions de vie des gens qui m’entouraient sans l'avoir jamais exprimée et ordonnée, qu'à mes propres conditions de vie dans ce que je crois avoir été un vrai terrain, inconfortable au regard de mes conditions d'origine en région parisienne (le long terrain tahitien qui s'ensuivit s’apparentait plus à la « Côte d'Azur » pour citer l'expression de Paul Ottino). Nous nous étions de ce fait là un peu rapprochés. Non seulement ils étaient différents de moi, mais ils étaient aussi des homologues, des alter ego. Vu d'Ambatozavavy ou d'Ampasimena et non depuis les salles de cours de Nanterre, le « relativisme culturel » me laissait par beaucoup d'aspects pour le moins songeur.

L'un de mes souvenirs les plus vivaces fut de revenir à Tananarive où j'étais si bien accueilli, après quelques mois d'un régime sans eau courante ni électricité, et d'allumer la lumière, en une simple pression sur un bouton ; puis de tourner un robinet pour que l'eau jaillisse sans autre formalité. Ce geste si banal, je le trouvais ce jour là proprement miraculeux. Cette eau 
là, il me fallait aller la chercher sur quelques centaines de mètres dans des jerricanes de 25 litres, et c'était déjà assez sportif ; et encore le village d’Ambatozavavy était miraculeusement pourvu d'une adduction, dont je ne sais toujours pas d'où elle provenait.

Ce n’était nullement le cas de tous les villages de la côte à palétuviers de Nosy Be, et ce n’était jamais le cas dans le splendide isolement de ce que l'on appelait la « Grande Terre » (Antagnibe, c'est-à-dire le continent), au Sud d’Ambaliha. Là on sentait bien que les hommes étaient seuls dans une vaste nature infiniment plus grande qu'eux, et que la seule marque qui reliait leur paysage à des centres de population était cette unique piste désormais goudronnée mais à ce moment là cahossée de trous de sable, dont la trace se perdait, qui s’enfouissait dans les cacaoyères pour se transformer en simple chemin, si bien qu'on se demandait si on était bien toujours sur la « nationale ». D’ailleurs c’était ce qu’on appelait lalabe le « grand chemin ». La route était aux yeux des paysans malgaches un objet si exotique et pour tout dire si étranger qu’on ne la considérait pas toujours comme un bien commun, l’un de ces biens qui, tel « l’intérêt général », appartiennent « à tous et à personne » mais, dans ce cas, plutôt à personne en particulier. C’est ainsi qu'un soir, dans un paysage de lagunes sableuses où se dessinait un radier de bois, je discernais une silhouette penchée sur le radier ; en m’approchant je vis un vieil homme tout noir et habillé de toile blanche, (probablement un Makoa, c'est à dire un descendant d'esclaves) qui ramassait tout simplement les gaulettes de bois posées sur les traverses. «Akory dadilahy » lui dis je par la fenêtre après qu’il m’eut laissé passer « mangala ala maiky é », ( « salut grand père, on ramasse du bois mort ?»). C’était ainsi que l'on saluait quelqu'un dans des espaces non socialisés comme la forêt ou la pleine nature, de manière à ne pas être suspecté d’intentions agressives. Et lui de répondre impertubablement «ia é ! mangala ala maiky é ! ) et de repartir au loin, son fagot de radier sur l’épaule. Le vieil homme ignorait sans doute qu'il contribuait ainsi, même un peu, à interrompre le trafic alors régulier, désormais incessant, de 404 « goelettes » qui relie toujours 


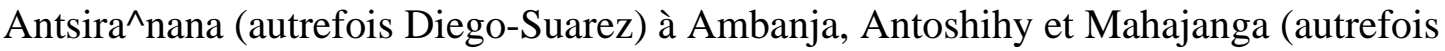
Majunga). Pour ma part, je sentais confusément dans l'attitude de l'homme quelque chose qui ressortait de l'incivilité; mais encore aurait-il fallu, me disais je aussi, qu'il aie lui-même à en tirer profit de cette route exotique, sans doute faisait il partie de la « pauvreté cachée » à la Robert Chambers. Je l'ai vu s’éloigner vers la forêt dans la lumière déclinante du soir, sa silhouette s’est amenuisée. Je ne l'ai jamais revu, et je crains bien qu’il soit mort depuis. Je crois que c'était un homme assez drôle.

Je me suis toujours souvenu aussi de la rage de dents de la tante paternelle Vao, que l'on appelait respectueusement « dady » (grand-mère) ou bien Ny angovavy « La tante paternelle » avec un grand $\mathrm{N}$ ou un grand $\mathrm{L}$. pour cette bonne raison qu'outre d'être une adorable vieille dame elle était la dernière détentrice des droits fonciers du groupe résidentiel. Un jour de Février 1971 je crois bien, la tante paternelle eut si fort mal aux dents qu'elle commença à se plaindre, puis à s’allonger sur la minuscule couche de sa minuscule case. Ca ne lui ressemblait pas. La visitant je vis que sa joue était entièrement enflée et je lui dis qu’il vaudrait mieux aller à l’hopital, à une quinzaine de kilomètres. Il y eut des conciliabules, mais la tante paternelle resta de longs jours sur son lit de douleur pendant qu'on lui faisait boire des tisanes de plante les unes après les autres.

J'étais si naif que je croyais à une épreuve imposée par de mystérieuses logiques : la tante paternelle était si spécifique qu'elle n’aurait pas pu être soignée par une autre médecine que celle de ses pairs. Je crains que la vérité eut été plus prosaique, et en tout cas différente : la tante paternelle Vao n'avait pas les moyens d'aller à l'hopital, et les conciliabules tenaient à savoir qui allait donner quoi, dans la situation impérative où tout le groupe devait faire d’indéfinis aller et retours à « Lapointy » pour l'accompagner. D’ailleurs, les gens avaient peur de l’hôpital, selon des raisons parfois parfaitement compréhensibles et parfois 
mystérieuses $\left({ }^{4}\right)$. Je regrette toujours, rétrospectivement, de n’avoir pu régler la situation plus tôt à grands coups de petits billets de banque, en ayant à décharge que je ne comprenais pas la situation et surtout que la situation squelettique de mon compte en banque pouvait difficilement affronter une telle option. Ces gens là n'allaient pas, tout simplement, à l’hôpital ; ils souffraient sur place, et certains mouraient sur place, point à la ligne. « C'est dans un char à bœufs, s’il faut parler bien franc », c'est dans un char à bœufs « poussé par les amis, tiré par les parents » que la tante paternelle Vao fut convoyée à un taxi « brousse » qui faisait des réductions si on se rapprochait de son principal point de ralliement.

Mais pour notre propos, et plutôt pour le mien, elle fut quasi instantanément soulagée par quelques piqures d’antibiotiques Je découvrais que même ces constructeurs baroques d’opéras familiaux sur la mort que sont les Malgaches avaient eux aussi un urgent besoin d’antibiotiques ; il faut dire que j’avais déjà guéri une infection au pied mais pas au pied de n’importe qui, celui précisément d’une guérisseuse, simplement en lui appliquant de l'auréomycine et en lui demandant de laisser son pied au sec autant que possible, ce pour quoi elle m’avait manifesté une vive reconnaissance $\left({ }^{5}\right)$

La bannière du relativisme culturel sous laquelle avançaient beaucoup d'anthropologues des années 1970 me paraissait à moitié en lambeaux. Au prétexte que les Sakalava du Nord pensaient indéniablement le monde d'une manière spécifique, que certains se souciaient fort peu d’être riches ou de mourir jeunes, on ne pouvait avancer qu’ils ne préféraient pas en général, tout comme nous, guérir d’une rage de dents ou d'un abcès au pied, vivre plus longtemps dans des conditions physiquement moins éprouvantes, bref accéder à une

$\left({ }^{4}\right)$ Voire le livre si pertinent publié par Yannick Jaffré et Jean-Pierre Olivier de Sardan chez Karthala, concernant les hopitaux d'Afrique de l'Ouest. (2003)

$\left({ }^{5}\right)$ Il est intéressant de noter que la seule mention de cette anecdote a provoqué l'ire d'une étudiante en anthropologie d'un DEA d'Université française, sans doute pour l'irrespect qu'elle était censée manifester envers 1) les guérisseuses non patentées ? 2)les guérisseuses exotiques ?. 
configuration de valeurs universalistes que l'Occident industriel avait petit à petit bricolées, souvent inintentionnellement $\left({ }^{6}\right)$. C'est ce que noterait Amartya Sen en 1988 en discutant l'extension du concept de développement, dans un article fondateur de son fameux travail sur l’Indice du Développement Humain: « »Le problème de l’hétérogénéité des valeurs est certainement un problème sérieux, mais il n’est nullement absurde de penser que les points d'accord sont très importants » $\left({ }^{7}\right)$ Et d'ailleurs, cette question atteignait par certains côtés l'activité anthropologique elle-même, pour de multiples raisons. Tout d'abord parce que si relativisme culturel il y avait vraiment, alors l’anthropologie pratiquée entre personnes de langues différentes était impossible ; c’eut été comme parler à des Martiens sans savoir s’ils savaient ce qu'était que « parler ». Entre personnes de la même langue, le problème ne faisait que se reproduire à l'infini, mais en plus petit si l'on veut. Le relativisme culturel de « L'anthropologie à domicile » pour citer une expression de la revue l'Homme faisait par exemple communiquer une anthropologue et les cadres d'une entreprise : en cas de relativisme il eut alors fallu que l'anthropologue fût elle-même un cadre, pour y comprendre quoique ce soit. «Qui est l’Autre » s’est demandé fort justement Marc Augé (1987). Pour ce qui me concerne, je n’ai jamais pensé que le fait que quelqu’un pense différemment de moi en fasse un « Autre » irréductible, et je n’ai jamais éprouvé le moindre tracas, sur le fond, à pratiquer cet étrange travail de médiateur « réaliste ». L’Autre était un peu de moi-même et j’étais un peu l’Autre, et il en était ainsi depuis que le monde était monde -ou, plus exactement depuis que les bébés apprenaient que quelqu’un s’appelait Maman, baba ou JeanPierre, c'est-à-dire depuis que les bébés avaient « découvert le langage que leurs parents comprennent », pour citer Jean Piaget.

\footnotetext{
$\left({ }^{6}\right)$ Baré 1987

$\left.{ }^{7}\right)$ « The problem of value-heterogeneity is undoubtedly serious, but it is by no means absurd to think that the actual extent of agreement is indeed quite large ». "The concept of development” in Chenery (H.) and Srinivasan (T.N.) Handbook of Development Economics, Vol.I, Elsevier Science publishers B.V., 1988:23)
} 
Mais la notion de relativisme culturel en vigueur en anthropologie me paraissait jeter le trouble sur la discipline par un autre biais, s’agissant de « développement ». Car quand même, ce qui avait créé l'anthropologie institutionnelle, c’était une histoire spécifique, celle de l’Occident euro-américain, au travers de laquelle des ressources dont il ne m’appartient pas de commenter la modestie avaient, par de complexes médiations sur lesquelles l'anthropologie classique ne s'appesantissait guère, créé des emplois publics comme ceux de coopérants militaires ou civils à l’Université de Madagascar, et même des chaires au Collège de France. En ceci je ne crois pas pour autant que la civilisation euro-américaine ait inventé quelque chose de vraiment nouveau ; simplement, elle l'avait domestiqué, puis institutionnalisé. Si je me suis si bien entendu par exemple avec des chroniqueurs paysans du Nord de Madagascar, c'est qu'ils constituaient eux-mêmes de sortes d'anthropologues spontanés (comme les Indonésiens m’a-t-on dit), et que les questions de parenté ou de définition des charges politiques constituaient des embrayeurs spontanés de la conversation. Ils avaient instantanément traduit mon travail : j’étais « un ramasseur de catégories claniques « (ampangala firaza^nana). Et si je voulais expliquer que je faisais de la statistique sur les familles du Nord cela s’appelait « mélanger tout ça » (mampiharo jiaby), ou plus littéralement « faire que tout ça se mélange » (miharo).

Il court de par le monde une foule d’anthropologues « sauvages », au sens de la Pensée Sauvage de Claude Lévi-Strauss. En ceci l’anthropologie est en quelque sorte universelle, et n’appartient donc à personne en particulier. Mais s’agissant d'anthropologie institutionnelle, si l'on admettait d'ores et déjà que le développement relève d'une forme de l'intervention publique, l'anthropologie n’est pas seulement la critique patentée du développement : elle en est aussi la fille, notamment dans ce qu'elle porte de message universaliste. Le relativisme culturel est lui-même relatif. 


\section{Rencontres avec des économistes}

La singularité n’étant pas un principe d'évaluation -et donc encore moins un principe d’autoévaluation- je crois avoir fait partie d'un tout petit nombre d'anthropologues à avoir entamé un dialogue direct avec des économistes, tout d'abord en France, notamment avec cette sorte d'économistes qui disent se préoccuper d' « économie du développement », puis par la suite aux Etats-Unis notamment $\left({ }^{8}\right)$. Bien que la « pluri-disciplinarité » eût été à l’ordre du jour, cette démarche n’allait pas sans difficultés, comme en témoignaient quelques réunions où j’étais accueilli courtoisement, mais comme un animal un peu bizarre, dont on ne savait trop que faire. J'eus même droit à ce qu'on taxe ma nouvelle demande de rattachement de " problème Baré ». Ceci tenait moins, je crois, à ma personnalité propre ou aux lourds habitus disciplinaires, qu'à la perplexité totale où se trouvaient nombre d’économistes français devant ce qui serait l’anthropologie en général. L’un disait s’intéresser à l'anthropologie, mais c'était simplement parce qu'il avait fait des enquêtes « de terrain », en parlant directement à des gens ; d'autres pratiquaient ce qu'ils appelaient l'anthropologie économique, qui relève plutôt à mon sens des animaux hybrides ni chair ni poisson. Il faut dire, symétriquement, que les économistes étaient souvent bombardés par les anthropologues de critiques pour leur ethnocentrisme- critiques dont on a vu à quel point il fallait les spécifier- ou, à tout le moins, de sempiternels attendus sur la diversité des organisations humaines qui les laissaient dans un désarroi compréhensible, puisque tel n’était pas leur propos ; ou bien, si c’était le cas, ils s’étaient transformés en sociologues plus ou moins performants sans le savoir. Au fond ce dont traite l'économie, c'est des problèmes

$\left({ }^{8}\right)$ Parmi ceux-ci je ne connais que le nom de Nicole Sindzingre du CNRS, membre dès ces années là du laboratoire d'économie du développement de Philippe Hugon à Nanterre. 
d'allocation des ressources rares, un processus dans le cadre duquel les institutions humaines jouent certainement un rôle central, mais à ne se préoccuper que de la diversité des institutions on oubliait les ressources elles mêmes. Dans le cas du « développement », le fait qu'une politique de soutien des prix à la production agricole concerne une société de de fermiers catholiques de Lozère, d'adorateurs de Vishnu ou de conteurs d'un certain Renard pâle, producteurs de mais, de riz, ou de mil n’offre en effet, de prime abord, aucun intérêt pour l'économie du développement, et non sans raison pourrait-on ajouter : il n’empêchera jamais un prix à la production d’être un prix à la production dans les trois cas. On ne voyait donc pas, symétriquement, à quoi pouvait ressembler l’ainsi nommée » anthropologie économique » mise en cause ici, avancée là, y compris par l’un de ses fondateurs en France, Maurice Godelier (1974 par exemple) : s’il était une anthropologie des systèmes de production, de distribution et de mesure des biens, c'est que ces systèmes connaissaient une variabilité constitutive du fait culturel ; dans ce sens cette « anthropologie économique » n’était autre qu'une anthropologie tout court.

Mon idée était assez analogue mais beaucoup plus circonscrite. Une approche anthropologique de terrain, fondée sur les énoncés des acteurs, consacrée aux institutions mêmes chargées de l'acheminement des ressources rares de par la planète -c'est-à-dire au « développement induit »- apportait des vues plus réalistes sur la constitution finale de ces ressources ; mais simultanément elle étendait à des appareils administratifs souvent vus spontanément comme purement techniques et fonctionnels la qualité d’objets anthropologiques au sens de Sahlins (1976), c'est-à-dire d'organisations porteuses de « schèmes symboliques déterminés qui ne sont jamais les seuls possibles ». En général, une démarche très « appliquée »-dirigée par un enjeu social particulier- ne paraissait nullement contradictoire avec une démarche plus générale, peut-être porteuse de visées théoriques plus 
larges. Vues sous cette angle, des institutions comme la Banque mondiale, telle ou telle de ses Vice-Présidences et de ses services divers et variés, la Société d’Aménagement Foncier et de Développement Rural de la Réunion ou bien la Banque de Développement Economique de Tunisie apparaissaient, de manière réaliste me semble-t-il, comme d’étranges créations de l’esprit humain, exactement au même titre qu’un rituel funéraire sakalava.

Ainsi en Tunisie, une ligne budgétaire d'aide à la création de petites entreprises serait créée par une banque multilatérale dans le cadre d'un dispositif dont la complexité baroque n'avait rien à envier à d’autres créations humaines plus fréquentées par l'anthropologie classique : une « ligne pilote », un syndicat de banques locales en compétition, des interprétations diverses sur ce qu'étaient au juste des entreprises « intenses en travail », des techniciens obéissants, ou perplexes, ou agnostiques, ou indifférents, comme les destinataires du prêt euxmêmes. Elle aboutirait à des entreprises beaucoup plus intenses en capital que celles prévues au départ; mais s'il en était ainsi, c’est que les critères d'accession au prêt et donc le dispositif administratif et bancaire avait fini, en évoluant au cours des années, par éliminer de lui-même les entreprises les plus conformes à ce qui était souhaité ( des entreprises « intenses en travail »). Ainsi encore, dans le Pacifique insulaire anglo-saxon, les dispositifs d'accès au même genre de soutiens utilisaient quasi exclusivement la langue du « business » angloaméricain, éliminant de fait les entrepreneurs monolingues; on avait oublié, comme cela est si fréquent dans le monde «normal », l’existence de langues étrangères. Ainsi toujours dans l’île créole francophone de la Réunion, la considérable réforme foncière entamée dès les années 1960 avait bien constitué une couche moyenne d'agriculteurs, en améliorant ainsi le sort d' une foule de petits métayers miséreux mais en laissant de son propre aveu $30 \%$ de ses « clients » sur le bord du chemin, qui finissaient en balayeurs intermittents des communes. Bien qu'elle fût éminemment utile et même indispensable à la collectivité -comme on le voit lors des grèves des éboueurs ici ou là- cette activité était fort mal rétribuée, alors que des 
producteurs de canne hautement subventionnée, à juste titre, par l’Europe y trouvaient un revenu souvent plus décent.

Dans aucun de ces cas, on ne pouvait rapporter ces résultats mitigés (à mon sens inéluctablement mitigés) à la méchanceté ontologique de tel ou tel acteur. C’était bien triste par certains côtés, ç’aurait été infiniment plus simple de « trouver le coupable ». En ces années là, en effet, on pouvait se demander si certains sociologues n’avaient pas «trouvé l'adresse du Diable » pour citer l'expression d’un ami économiste, qui se serait située au 1818 H St NW, à Washington, au siège de la Banque mondiale. Ces résultats devaient être rapportés à l'organisation de « dispositifs » institutionnels (et donc sociaux), c'est-à-dire à des liens organiques entre acteurs, qui agissaient sans doute inconsciemment comme des Homo aequalis à la Louis Dumont, dispositifs dont la réforme était sur certains points criante de nécessité, mais dont la globalité m’aurait laissé perplexe; ils étaient hétérogènes les uns aux autres et relevaient en ceci d'une approche anthropologique au sens strict, qui mettait au jour des fonctionnements, (évidemment « interactifs ») qui échappaient à chaque acteur - et donc, dans le cas de chefs de service, à chaque personne- pris isolément). En ceci je ne crois pas pour autant avoir soutenu de ces positions dites « durkheimiennes » où ce qui serait le collectif aurait pris le pas sur ce qui serait l’individu. Même dans ces univers bureaucratiques, l'hypothèse de la liberté sartrienne dans un monde sans Dieu (dans un monde peut-être sans Dieu), et donc de la liberté individuelle, pourvue d'une conscience, me paraissait infiniment plus plausible; mais elle n'était pas pour autant contradictoire avec l'existence de contraintes institutionnelles. Comme je l'ai souvent fait remarquer par la suite, des gens aussi puissants que des Vice-présidents de la Banque mondiale pouvaient aboutir à des résultats opposés selon leurs propres capacités d'autonomie: si l’un, par exemple, ne voulait pas prendre en charge les revendications des ONG sur le fameux barrage sur la Narmada en Inde, d'autres 
avaient «fait remonter« l’information sur le désir de paysans guatémalteques réfugiés de reconstruire eux-mêmes leurs maisons, au lieu d'avoir des maisons en kit. $\left({ }^{9}\right)$. J'en avais depuis longtemps été prévenu par des amis du Nord Malgache lorsque je leur parlais de leurs « règles » (didy). Ils me répondaient parfois que ce que suivaient les gens en général c’était en fait leur sitrapo, leur « inclination », leur « liberté », ou plus littéralement leur « selon -leursentrailles » (sitrana-fo), mais en oubliant du même coup le champ intellectuel et donc sociologique où s’inscrivait nécessairement cette liberté là; en ceci ils semblaient assez étonnamment épouser les apories de l' »individualisme méthodologique », vu l’univers hiérarchique et holiste dans lequel il baignaient, mais il y avait quelque « véridicité » dans leur attitude ; certains se rendaient aux rituels royaux, d'autre pas ; etc.

On trouve je crois chez Spinoza que « la liberté c’est la conscience de la nécessité ». Ceci rencontrait presque parfaitement, en tout cas, ce qu’Albert Hirschman théoriserait par la suite dans les termes d'exit et de voice dans les institutions, l’opposition entre la « défection » et la « prise de parole » (1972). Je voulais me contenter de raconter de manière aussi véridique que possible, en ayant la secrète satisfaction de mettre au jour des faits, généralement parfaitement banaux, mais là où on ne les avait généralement pas cherchés.

\section{Le développement comme catégorie d'un système sémantique ; les théories du développement comme mythe d'origine}

Pour être fidèle à la démarche proposée ici, il me faut revenir à un travail qui a sans doute été originel dans cette partie de mon itinéraire, et qui m’avait été demandé vers 1985 par mon ami Gérard Lenclud, alors qu'il était rédacteur en chef de la revue Etudes Rurales et moi-même

$\left({ }^{9}\right)$ PARTRIDGE 1995 cité in Baré 2001 b 
membre d'un comité de rédaction où j’ai du siéger une bonne douzaine d’années. Il était très attentif aux « nouveaux objets » qui se dessinaient alors en anthropologie, un mouvement qui tenait autant d'après moi aux difficultés d'accès aux terrains classiques, perturbés par une géopolitique déjà un peu plus folle que d'habitude, qu’à l'extension ontologique d'une nouvelle curiosité tous azimuts. Il était donc assez naturel qu'il me propose de rédiger une sorte de texte résumant les orientations générales du programme que je commençais à me donner, tout en manifestant devant moi son étonnement sur le fait qu’il « était possible de faire ce genre de choses à l'ORSTOM »; ceci montrait au moins le genre d'image qui courait dans le monde académique sur ce qui était déjà un EPST, à l’égal du CNRS.

Vu l'estime que je porte à Gérard j’ai pris ce projet très au sérieux. Il en résulta cependant un long été studieux où j’ai appliqué à une quantité assez importante de textes le principe de l'anthropologie de terrain d'Evans-Pritchard selon lequel « l'informateur a toujours raison »; la première démarche qui contient toutes les autres était de trouver d’abord une définition du concept de développement qui se tienne plus que les autres, ou bien qui ne se tienne pas du tout. Mais la quantité étant si grande et la redondance aussi, et mes bagages d'une taille forcément limitée, je me suis tenu à ceux des textes que je trouvais un peu arbitrairement fondateurs pour un exercice de définition. Ceux de l'économie du développement -ainsi du manuel fascinant de rigueur que Patrick Guillaumont, fondateur du CERDI de ClermontFerrand venait de publier, de Everett Hagen parmi mille exemples de livres intitulés « Economie du Développement », puisque la notoriété publique me faisait savoir que le concept de développement était d’abord économique, de cette étrange sorte d’économie qui s’était institutionnalisée à la fin de la deuxième guerre mondiale ; ceux de Simon Kuznets sur la croissance économique, de Rostow, d’Arthur Lewis, Kindleberger et j’en passe. Ceux d’historiens et économistes aussi - et c'était peut-être plus inattendu- qui traitaient de la révolution industrielle en Europe ; puisque la notion de développement procédait de 
l'économie politique, qui s’était progressivement transformée au cours de cet évènement majeur dans l'histoire du monde où l'Occident vit émerger massivement la fabrique de Marx, l'industrie, la production de masse, l’augmentation de la productivité agricole, la statistique. Ceux donc de Fernand Braudel (« ne domine pas le monde qui veut », à propos de l’Angleterre), Hobsbawm, Norman Rich, Paul Bairoch et j’en passe, bien sûr. L’œuvre d’Amartya Sen était à peine connue des non spécialistes, et l'admirable livre de Jean-Claude Perrot sur «une histoire intellectuelle de l'économie politique » n'avait pas encore vu le jour ; il s’arrête d'ailleurs à la fin du XVIIIème siècle.

Au sortir de cet exercice assez austère, en tout cas aussi consciencieux que possible, je me suis trouvé dans une situation finalement assez familière à celle d'un anthropologue « classique » lorsqu’on lui explique les mots de la langue. Il n’était pas intellectuellement possible de différencier la notion de développement de celle de croissance économique, dont elle était censée pourtant se distinguer; pas plus qu’il n’était possible de définir le terme du Nord malgache kabaka, qui désigne tous les accompagnements culinaires du riz dans les repas (poulet, bœuf, coquillages, poissons), indépendamment du riz lui-même $\left({ }^{10}\right)$. Le développement, croyais-je, pouvait être défini comme « une sorte de cadeau Bonux que l’on trouve un peu par hasard dans un paquet de croissance économique ». En somme, le terme relevait d’une de ces « catégories locales » qui m’avaient tellement fasciné lorsque j’avais entamé un travail d'anthropologue. De ce fait, tout ce qui concernait le développement (théories, pratiques, jusqu’à la plus minuscule des ONG) se trouvait irrémédiablement marqué par cette ambiguïté de définition. Cette constatation, longuement argumentée je crois in Baré 1986, a forcément nourri à nouveau une attitude qui traitait de l’affrontement entre « théories » du développement comme d’une pratique complètement vaine ; alors que, comme

$\left({ }^{10}\right)$ On trouve chez Sen : « The close link between economic development and economic growth is simultaneously a matter of importance as well as a source of considerable confusion” (1988: 12). 
je l'ai déjà dit, la dimension récurrente de l'intervention publique -qui contenait l'aide au développement comme une sorte d'enfant non reconnu- me paraissait une dimension éthiquement indispensable, si l'on voulait absolument me demander « d'où je parle », selon l’expression pratiquée de nos jours.

\section{Enquêtes de terrain}

Je crois en ces matières que l'enquête directe auprès d'administrations, nationales, multilatérales, peut s’avérer d’une grande utilité pour les collectivités en cause, pour peu qu'elle soit plus synthétique qu’analytique, sans parler d’apporter des matériaux à cette discipline parfaitement floue, si j'ose dire, qu'est la sociologie ou l'anthropologie des institutions publiques. J’ai souvent entendu dès 1986 qu'il était difficile d'approcher des administrations qui sont, parait il, des mondes fermés et défendus :, et encore en 2004, une personnalité nommée d’une instance d'évaluation me faisait part à ce propos d'un léger étonnement mêlé d'effroi. Pourtant, jamais personne ne m’a fait le même genre d'objections à propos de l'approche de paysans malgaches ou de diacres tahitiens ; ce n'est pas si facile non plus.

Les équations personnelles étant ce qu'elles sont , je suis parti vers ces enquêtes dans un état d’esprit exactement analogue à celui d’enquêtes antérieures plus « classiques ». La curiosité, la retenue du jugement, la nécessité d’une écoute aussi attentive que possible des énoncés des personnes et surtout de leurs associations, tant au sens linguistique (leurs associations verbales souvent porteuses de logiques implicites) qu'au sens de leurs associations avec d’autres personnes placées dans le champ de leur action, c'est-à-dire bien sûr de leurs « interactions ». Au titre adventice, je peux témoigner à nouveau s’il en était besoin que les hiérarchies administratives ne fonctionnent pas comme le suggèrent leurs organigrammes, que 
les « services « et les « directions » sont habitées de choix et de tactiques, bref de liberté par certains côtés ; et que la « rationalité limitée » de March et Simon propre aux organisations à nouveau commentée par Raymond Boudon dans un beau livre (2003 )est certainement une hypothèse plausible, à moins que ce ne soit la seule.

Pour que ces enquêtes aient lieu, il fallait toujours un commencement, exactement comme dans les enquêtes de terrain classiques, c'est-à-dire une introduction pour que les enquêtés soient amenés à communiquer dans un cadre aussi paisible que possible, qu’on ne me méprenne pas pour un espion du KGB ou un évaluateur sous-marin du ministère de l’Economie. Simultanément, je ne pouvais pas prétendre m’intéresser au fonctionnement d'une ligne budgétaire ou d'une réforme foncière pour les beaux yeux de la science, ou comme je le disais aux chroniqueurs sakalava pour « apprendre l'histoire aux enfants » bien que ce n’eut pas été complètement inexact dans le cas qui nous préoccupe, et tout à fait plausible dans l'autre. Je m’ appuyais d'abord sur la référence institutionnelle (l’ORSTOM est intéressé par ce que fait le gouvernement tunisien pour les petites entreprises, ou la SAFER Réunion pour la réforme foncière), ensuite sur ces introductions qui demandèrent au début beaucoup de courage et de foi auprès de mes garants qui avaient eux aussi leurs contraintes, introductions qui avéraient cette vocation dès lors un peu officielle; enfin sur l'expression d’un intérêt personnel (au sens d’être « intéressé par ») qui n’était pas feint, bien que je me sois aussi beaucoup ennuyé. Mais c’est la rançon du terrain parait il. ( ${ }^{11}$ ) Je dois la première de ces introductions à l'économiste Jacques Charmes, spécialiste de la mesure du travail informel, auprès du Ministère des Affaires sociales de Tunisie, alors qu’il était responsable d'une unité de recherche $\left({ }^{12}\right)$. Assez étonnamment au vu de son profil intellectuel et de ses fonctions de l'époque (il travaillait au service de Coopération de l'INSEE

$\left({ }^{11}\right)$ Dans Lettres à la fugitive, (1986) un livre injustement moqué, l'anthropologue Emmanuel Terray évoque avec une criante vérité les longues heures passées chez les Abron de Côte d'Ivoire à lire la Guerre et la Paix de Tolstoi dans le caquètement des poulets, en attendant que quelque chose se passe.

$\left({ }^{12}\right)$ Il n'est évidemment aucunement responsable des opinions qui pourraient apparaître dans ce texte. 
à Paris, après de longues années tunisiennes) il se déclarait sincèrement intéressé par ce qu’il appelait l’approche anthropologique, c'est-à-dire, à vrai dire, l’interview systématique de petits ou de micro-entrepreneurs. Le caractère « anthropologique » de cette approche apparaissait plus précisément, au sens retenu dans ce texte, dans ses remarques critiques sur le fait que les comptabilités nationales tendaient à penser que les petites entreprises du secteur formel ignoraient ce qu’était la comptabilité. Pour lui, il s’agissait bel et bien d’un autre type de comptabilité que celle qui décline le « capital fixe », « l’actif circulant » et autres « fonds de roulement », mais qui aboutissait de toute évidence à des résultats parfaitement honorables ; ces entreprises là se développaient, mouraient, s’associaient, subsistaient, selon ce qu’il n’appelait pas des logiques sémantiques différentes à propos de la valeur, mais c’était bien de cela dont il s’agissait, et ces entreprises là étaient bien fonctionnelles, et étaient bien des entreprises. Je regrette un peu, rétrospectivement, que cet aspect de nos conversations n'aie pas donné lieu à une recherche commune sur un thème qui me paraissait extensible à de nombreuses situations propres à ce que l'on peut appeler l'économie populaire, celle des « ménages » par exemple, c'est-à-dire sur un thème pertinent au sens le plus scientifique possible. Mais il est vrai que dans le cas Tunisien je ne parlais pas l'arabe et que c'était une condition sine qua non de ce genre d'exercice, bien que le fossé entre les conceptions institutionnelles et les conceptions populaires soit tout autant constatable -bien que moins évident peut-être- dans la même langue, comme le Français par exemple Cette remarque de Charmes a sans doute constitué le déclencheur d'un autre aspect de mon travail concernant les problèmes de traduction dans l'aide au développement, si l'on admet que les théories mais aussi les institutions de développement sont informées par les langues dans lesquelles il s’exprime. Dans le cas du Pacifique polynésien mes conclusions seraient moins nuancées que les siennes, les entrepreneurs tahitiens par exemple ne pouvaient pas parler à des institutions publiques s’ils ne parlaient, par exemple, que tahitien (1992). 
Ces premières enquêtes eurent aussi pour effet de me montrer qu'une démarche s’intéressant sincèrement au travail des experts et gestionnaires du développement pouvait susciter leur intérêt. Ce qu’il faut bien appeler mon « complexe » d’anthropologue (ou bien d’être humain, simplement ?) tenait à ce que les gens que j’approchais me prennent pour quelqu'un d’incompétent, et qu'il ne valait donc pas la peine de me parler. Fort heureusement j'étais pourvu d'une culture économique minimale, et, en fait, je crois bien qu'elle leur suffisait largement, bien que je ne me sois jamais mêlé de fournir des solutions économiques -et d’ailleurs on ne me l’a jamais à proprement parler demandé. Après toutes ces années je me demande toujours d'ailleurs, sans doute avec une arrogance je l'espère excusable, si les dizaines d'experts du développement que j’ai rencontrés ne m’ont pas parlé en fonction d’un socle minimal de propositions empiriques au regard desquelles la distinction entre les écoles et interprétations « néo-classiques », « institutionnalistes », « néo institutionnalistes », « keynésiennes » « néo keynésiennes » de l’économie ne constituent pas un ensemble de corpus aussi peu « réfutables » et « prouvables «, pour parler comme Karl Popper, que ceux dont se réclamaient les jansénistes et les jésuites au XVIIIème siècle en opposant la « grace efficace » et la « grace suffisante ». En fait, pour eux tous sans exception, il fallait de la dette mais pas trop (ce « trop » là était criant dans les années 1980-1990 dans beaucoup de pays du « Tiers Monde ») ; il fallait des entreprises privées et non des entreprises publiques pléthoriques, mais l’Etat devait s'en mêler un peu (comme dans le cas de la Mission sur l’Emploi Industriel de Tunisie) ; les taux de change devaient être réexaminés, et les monnaies dévaluées ou réévaluées les unes contre les autres jusqu’à un certain point; en somme, dès qu'ils étaient pris dans l'action, la grande majorité militait en faveur d'une gestion moyenne de père de famille d'âge moyen -à l'exception sans doute de certaines franges extrémistes, comme ces thatchériens hystériques de l’OCDE avec qui j’avais diné 
chez des amis et qui m’avaient quitté en me souhaitant " good luck ", après quelques sorties cyniques sur le fait que « ces gens n’ont qu’à rembourser », (C’était l'époque de « I want my money back » de Margaret Thatcher). Ce sont hélas des spécialistes de ce genre de théologie qui tinrent le haut du pavé dans les institutions de développement des années 1980-1990, qui n’avaient pas prévu que leurs remèdes de cheval tuaient le malade « bien que l'opération ait réussi », remèdes qui aboutirent finalement à des programmes considérables de remise ou d'annulation de la dette et à des critiques cinglantes de l'économiste en chef de la Banque Mondiale lui-même, dix années après (Stiglitz 2002)

D’ailleurs traiter de projets d'aide financière auprès d'économistes, c’était immédiatement être économiste aux yeux de beaucoup d'entre eux, sans doute par la même magie du contact qui amenait les rois de France à guérir les écrouelles. En Tunisie l’un d'entre eux me demanda même, au vu de mon nom, si j’étais apparenté à Raymond Barre, ancien Premier Ministre français et auteur d'un très célèbre manuel d'économie politique. Dans leur grande majorité je les vis comme des gens sympathiques, travailleurs jusqu’à l'extrême, sincèrement dévoués à leur métier, et beaucoup plus distanciés vis-à-vis de leur activité qu’on ne le croit généralement, (pour certains la Banque centrale n’était qu’un vaste « magma » les sacro saints »taux de rentabilité interne » ou TIR relevaient de la « fiction »); mais aussi, hélas trop souvent, comme des gens très incultes sauf dans leur discipline, qui leur tenait exclusivement lieu de Weltanschaaung $\left({ }^{13}\right)$. Des personnalités, enfin, qui tenaient le monde vécu au bout d’une perche, du fait de la difficulté de relier leur place dans la chaîne de décision financière à l'état des régions où ils travaillaient, ce qui constitue une variante des épineux problèmes de causalité rencontrés en histoire, et du peu de temps que leur laissait leur charge de travail

$\left({ }^{13}\right)$ J’ai été frappé ainsi du nombre d'économistes qui méprisaient la musique comme une activité futile, ceci alors même qu'elle constitue de toute évidence une préoccupation majeure de beaucoup de gens par le monde. Le très intéressant témoignage de Robert Klitgaard, qui parvint à faire improviser des chansons douces-amères sur leur pays par les clients d’un café de Malabo, est un bon contre- exemple (1991) 
pour aller dialoguer longuement avec les personnes mêmes qu’ils étaient censées aider, petits entrepreneurs de la rue à Tunis ou à Suva, Rmistes de la Réunion, ou autres vendeuses à la sauvette de la bande de Gaza ; ce qui leur aurait permis d’après moi une vision de leur métier plus sereine, moins tendue et sans doute plus tragique. ( ${ }^{14}$ ) Si bien que l'ancien Président de la Banque mondiale nouvellement nommé déclara en 1996 qu’il souhaitait que les cadres fassent des séjours obligés dans les bidonvilles du Tiers Monde, ce qui suppose que ce n’était pas le cas à l'époque. Mais ceci ne doit pas faire rentrer dans des transes démagogiques. Le contact n'est rien en soi : pour en dire quelque chose d'utilisable ou de lisible, il faut abstraire.

Il est peut-être surprenant que j’aie été amené pendant toutes ces années à un dialogue assez étroit avec l'anthropologue et sociologue Michael Cernea, responsable à l’orée de son itinéraire de la seule équipe d’anthropologie et sociologie de la Banque mondiale, qui s’étoffa rapidement par la suite, et plus tard responsable d’une Task Force sur le développement social lors de la considérable réforme des années 1996 sq. . C’était la seule démarche -mais elle était notoire- qui souhaitait réconcilier contraintes économiques et analyse sociologique (ou bien l'inverse), depuis l'intérieur des institutions du développement, et de la plus puissante d'entre elles. Je l’ai commentée longuement (1994, 1997 notamment), en général avec le grand assentiment de Michael (qui trouvait cependant quelques attendus irrévérencieux envers la Banque). La grande sympathie qu'elle m’inspirait était tempérée par ce qui m’apparaissait comme des points aveugles. Militant pour que la Banque mondiale « adapte les projets aux gens » et non l’inverse, cette entreprise considérable n’avait que fort peu considéré l'impact des formes organisationnelles sur ses recommandations. Certes le vaste champ parcouru à ces époques par l’équipe de Cernea amenait à des vues très pertinentes sur des domaines aussi

$\left({ }^{14}\right)$ Cette question est brièvement abordée in Baré 1991 
essentiels que la construction des routes ou l'ainsi nommée « foresterie »; et surtout sur le problème du déplacement forcé, originel dans son itinéraire, thème à propos duquel il voudrait bien faire figurer la version française d'une sorte de texte-bilan dans un livre collectif que je dirigerais (Cernea 2001).

Ceci dit, « adapter les projets aux gens » était une formule séduisante, mais on en voyait immédiatement les limites si on la traduisait par « adapter la Vice-Présidence des Opérations au piétinage des rizières malgaches par les bœufs ». En somme, si précieuse put elle paraître aux yeux de beaucoup de communautés du monde, et si critique eut il pu apparaître en vers son institution, l’anthropologie sociale de Michael n’avait pratiquement pas abordé ce qui semblait pourtant constituer l'essentiel de son propos, la relation entre formes institutionnelles des institutions de développement, et projets de développement.

Il est fort possible -voire même très vraisemblable- que cette forme particulière de l’intervention publique qu'est l'aide multilatérale au développement se soit définitivement stabilisée en une forme d’homéostase, d’équilibre instable mais indéfiniment reproductible, auquel cas il n’y aurait plus rien à en dire. Contrairement à des critiques radicaux, je n’ai jamais trouvé d’argument très pertinent pour supprimer la Banque mondiale, pour cette raison que vu la « mondialisation » des choses il faudrait très certainement en réinventer une. Pour autant, cet unanimisme logique ne dispense pas d'examiner des facteurs très spécifiques de l'institutitution. Ainsi des niveaux de salaire et d'indemnités, dont un ancien Vice-Président a décrit le caractère arbitraire au-delà d’un certain niveau convenable, dont il faut bien sûr reconnaître la nécessité (pour attirer les personnels qui autrement se caseraient ailleurs, dans les banques tout courts ou les multiples entreprises demandeuses d'experts). Sa question « estil absolument nécessaire de voyager en classe affaires », suscita un véritable tollé dans l'institution $\left({ }^{15}\right)$. Même si la Banque souligne la part comparativement minime des dépenses

$\left({ }^{15}\right)$ Voir Michael H. K. Irwin, ancien directeur of du Departement des Services de Santé puis Vice- Président du Personnel. http://www.cato.org/index.html, Cato Foreing Policy briefing 253. 
de personnel dans son budget de fonctionnement, (11\% estimés vers 2000), il n’était pas interdit de penser qu'un fonctionnement plus sobre était de nature à rapprocher son personnel des « gens » (people first) célébrés par l'équipe de Cernea. Mais ceci n’était rien au regard du fonctionnement même de la Banque en vue de l'approbation des prêts ou même des aides directes : pour un petit paysan c'était un peu comme parler à l'Empereur de Chine, si bien que les fonctionnaires de la Banque me parlèrent à plusieurs reprises de la Vice Présidence des Opérations comme d'une sorte de Politburo, ou de son fonctionnement «Kgébien » (de l'ex KGB soviétique). En somme si l'on en venait, assez inexorablement, à ce genre de sujets la Banque ne pouvait être vue comme l’institution fonctionnelle dont elle aimait à présenter l’image.

\section{Des « écoles » d'anthropologie du développement ?}

On pourrait peut-être me trouver me trouver « mal luné » si, après avoir considéré que le terme de développement n’avait aucune définition opératoire bien que ce soit une activité parfaitement repérable, je dirais la même chose de ce que j’ai appris progressivement s’appeler l'anthropologie du développement, et non sans surprise que l'on me tenait de ci de là pour l’un de ses représentants français. Il y a pourtant quelque cohérence dans cette attitude. Jean-Pierre Olivier de Sardan considérait récemment dans une sorte d'article-bilan qu’il « règne une grande confusion autour de l'anthropologie du développement » (2003 : 729) et c’est donc avec une indéniable logique (et un certain soulagement de ma part) que je n’y suis pas cité ni au mieux de ma connaissance dans aucun de ses travaux, même dans la plus minuscule note infra-paginale.

Ce constat d'une « confusion » cède vite la place à une approche « centrée sur l'analyse de l'imbrication des logiques sociales » entre des univers qui seraient « développementistes » et 
les autres (ibid. 742), ce qui réitère les positions d’un travail remarqué et souvent répétitif, centré en gros, sur le propos d’une sociologie qui serait « interactionniste » (par exemple 1995) ; une distance est prise avec l'un des inspirateurs de cette démarche, Norman Long (par exemple 1989), qui aurait été bienvenue d’après moi dès son livre connu de 1995 . Pour autant, finalement, « on ne distingue plus ce qui relève d'une aide externe et d'un développement endogène » (2003 : 748) ; autant dire qu’on ne voit plus dès lors où se niche « l'interactionnisme » pourtant revendiqué, à supposer que l'on puisse parler d'une sociologie « non interactionniste ». Sans trop enfoncer l'on ne sait quel clou on ne voit donc pas trop non plus ce que « la prise en compte des aspects manipulateurs et débrouillards du comportement », « d'un point de vue offensif ou défensif » (ibid.) pourrait bien apporter soit à la connaissance fondamentale fortement revendiquée par Olivier de Sardan, soit à une anthropologie appliquée à l’attention de « décideurs » ou de gestionnaires, lesquels en ont vu bien d'autres (comme le note étonnamment l'auteur lui-même, ibid. : 734 note 1); sinon, une tautologie sociologique de plus, faussement revendiquée à mon sens comme une avancée théorique. J'hésite d'autant plus à le noter vu la grande estime que je porte d’une part à la considérable activité de formation et de vraie coopération impulsée par Jean-Pierre Olivier de Sardan, et à certains de ses travaux -comme son article sur « la politique du terrain » (1995b).

Cette « anthropologie du développement » là, mi-revendiquée, mi-niée, m’a toujours paru relever plus de la conjoncture soulignée en son temps par Marc Augé à propos de « l'anthropologie de la maladie » (1986) que d’une discipline constituée par un corpus de propositions spécifiques et « poppériennes ». C’est certes, d'un certain côté, la position de l'auteur lui-même (par exemple 2003 : 730), pour lequel « l'anthropologie est (...) une science sociale empirique »(...) « les sciences sociales relèvent (...) d’une logique du 
plausible (...) et restent fortement contraintes par une recherche de véridicité empirique » (ibid.), toutes positions qui attirent fortement ma sympathie. Mais «l'anthropologie » ou « les sciences sociales » ce n’est ni «l'anthropologie du développement », ni « les sciences sociales du développement ». Dans ce cas pourquoi constituer le "développement" et le "changement social" comme "un domaine de recherche à part entière pour l'anthropologie et la sociologie" ? (1995a:5). La position purement nominaliste souvent adoptée par Olivier de Sardan selon laquelle «(...) il y a développement du seul fait qu'il y a des acteurs et des institutions qui se donnent le développement comme objet ou comme but» (1995 : 7) présente quand même une difficulté, parce que pendant ce temps là les mêmes gens ne sont pas en train de cultiver des fleurs, ou de conduire la locomotive vers Mombasa ; ils gèrent des crédits publics, en fonction de buts particuliers, comme l'augmentation du revenu dans les régions périphériques, la distribution d’eau potable ou la formation des cadres infirmiers.

Ce qu'il faut bien appeler cette abdication originelle m’a toujours semblé jeter elle aussi une « confusion » persistante sur un travail pourtant commenté urbi et orbi depuis une décennie, qui en explique d’après moi le caractère répétitif et assez indécis au bout du compte, du propre aveu de son auteur (ci-dessus). Combien de fois ai-je entendu que des projets de développement constituaient des « arènes » selon Norman Long, (c'est-à-dire des lieux intellectuels de compétition pour les ressources) qui se définissaient entre « intervenants » et « populations-cibles » qui sont « au contact » (1995: 55, souligné par moi). « La belle affaire que ces arènes » me suis-je souvent dit à part moi; mais il est en tout cas impossible, en toute logique, de limiter une analyse des projets de développement aux gens qui sont « au contact », parce que nombre d'acteurs concernés ne le sont pas : il en va ainsi des macro-économistes, administrateurs et autres chefs de division dont les projets évoqués sont pourtant ontologiquement dépendants, sans parler de gouverneurs de banque centrale, de secrétaires au 
Trésor et autres directeurs de caisses d'amortissement. Le fait que dans l'intervention de développement les acteurs concernés ne sont pas « au contact » peut d'ailleurs constituer au contraire un élément de définition de l’intervention publique elle-même.

Je ne peux pas avouer avoir toujours constaté des « arènes » autour de projets de développement, ni des « groupes stratégiques » pour parler comme Olivier de Sardan, sachant que ces projets sont eux-mêmes des créations de médiations institutionnelles plus lointaines. Par contre j’y ai toujours vu des sortes de « romans vrais » pour parler comme Paul Veyne (1971), c'est-à-dire des êtres sociologiques certes interactifs, dépendants de procédures souvent aléatoires de cognition -c'est-à-dire de catégories de la « pensée sauvage » au sens de C. Lévi-Strauss (1962)- et pertinents uniquement dans une durée donnée, relevant donc finalement de l'écriture de l'histoire. Il est possible que chez Olivier de Sardan ce tropisme vers le conflit soit du au caractère qui me semble assez spécifique, y compris économiquement parlant, du « développement rural » en Afrique de l’Ouest, qui a fortement nourri son itinéraire, sans parler de l'attention sans doute justifiée, mais trop exclusive à mon sens, qu'il a portée à l’ainsi nommée « Ecole de Manchester »

Bien sur, son réel effort de compréhension générale doit être salué pour ce qu’il est, mais j’aurais tendance à me sentir beaucoup plus proche de la démarche qu'il a poursuivie avec Yannick Jaffré dans ce beau livre sur la « médecine inhospitalière » sévissant dans la même Afrique de l’Ouest. « Raconter une histoire vraie » (Baré 2001) voilà un programme que beaucoup trouveraient rudimentaire à propos des politiques de développement, mais personnellement je le trouve aussi assez robuste, voire même ambitieux. Car il faut savoir ce que l’on veut : décrire plus globalement en indiquant des incompréhensions et des manques, ou sélectionner des aspects plus partiels susceptibles de théorisation ? Il y a là d’après moi un 
beau programme pour lequel les capacités descriptives de l'anthropologie de terrain -et donc la littérature réaliste ?- semblent tout indiquées. 


\section{REFERENCES BIBLIOGRAPHIQUES}

\section{AUGE (M.)}

1975 Théorie des pouvoirs et idéologies. Etudes de cas en Basse Côte d’Ivoire. Hermann, Paris, 440 pp.

1986 « L’anthropologie de la maladie » L’Homme, Paris, 97-98.

1987 « Qui est l’autre ? Un itinéraire anthropologique ». L’Homme, Paris, 103

\section{BARE (J.-F.)}

1987 "Pour une anthropologie du développement économique" Etudes Rurales, n 105-106, Paris, 267-295.

1991 « L’aide au développement est bien humaine. Propos d'étape sur une recherche » Chroniques du SUD nº, ORSTOM, Paris, 151-159

1992 « L’économie décrite en tahitien. Quelques remarques. L’Homme, Paris, 121. 143-163 1994 « Une pensée positive ? Anthropologie sociale et développement rural » L’Homme, Paris, 131, 129-136

1995 a (dir.) Les applications de l'anthropologie. Un essai de réflexion collective depuis la France. Karthala, Paris. 282 pp.

1995b «Le champ de l’anthropologie. De quelques indécisions » in Les applications de l’anthropologie. Un essai de réflexion collective depuis la France. Karthala, Paris, 27-48 1995 c « En quoi peut bien consister une anthropologie appliquée au développement » in Les applications de l'anthropologie. Un essai de réflexion collective depuis la France. Karthala, Paris, $141-178$ 
1996 Compte rendu de J-P. Olivier de Sardan 1995a in l’Homme 140, Paris, 178-181

1997a "Que se passe-t-il dans les politiques de développement ?" Introduction à Regards interdisciplinaires sur les politiques de développement. L’Harmattan, Paris, 9-17.

1997b " Le prêt et le résultat. Eléments d'une chronique de l'évaluation à la Banque mondiale". In Regards interdisciplinaires sur les politiques de développement., 49-73

2001 « L’évaluation de l'intervention publique comme exercice d'anthropologie historique » in J-F. Baré (ed.) L'évaluation des politiques de développement. Approches pluridisciplinaires. L’Harmattan, Paris, « Logiques politiques », 91-119

BOUDON (R.)

2003 Raisons, bonnes raisons. P.U.F. Paris 254 pp.

CERNEA (M.) (ed.)

1991 Putting People First. Sociological Variables in Rural Development. 2nd edition revised and expanded. The World Bank, Oxford University Press (traduction française revue et augmentée La dimension humaine dans les projets de développement). Karthala, Paris, 1998. 575 pp.

2001 « Risques d’appauvrissement et développement : un modèle pour la réinstallation des personnes déplacées » in J-F. Baré (ed.) L’évaluation des politiques de développement. Approches pluri-disciplinaires. L’Harmattan, Paris, « Logiques politiques » 175-231. 
GEERTZ (C.)

1983 Bali. L'interprétation d'une culture. Gallimard, Paris. 325 pp.

GODELIER (M.) (ed.)

1974 Une discipline contestée : l'anthropologie économique. Mouton, Paris -La Haye, 371pp.

HIRSCHMAN (A.)

$1972 \quad$ Face au déclin des entreprises et des institutions. Editions ouvrières, Paris

(traduction de Exit, Voice and Loyalty, Harvard University Press, Cambridge, USA.), 137 pp.

JAFFRE (Y.) et OLIVIER de SARDAN (J.-P.) (dir.)

2003 Une médecine inhospitalière. Les Difficiles Relations entre soignants et soignés dans cinq capitales d'Afrique de l'Ouest. Karthala, Paris 374 pp.

KLITGAARD

1991 Tropical Gangsters. One man’s experience with Development and Decadence in Deepest Africa. I. B. Tauris and Co Ltd, London and New York. 281 pp.

LEVI-STRAUSS (C.)

1962 La Pensée sauvage. Plon, Paris 380 pp.

LONG (N.)

1989 Encounters at the Interface. A perspective on social discontinuities in rural development. Wageningen, Agricultural University.

OLIVIER DE SARDAN (J.-P.) 
1995a Anthropologie et développement. Socio-anthropologie du développement et du changement social. Karthala, Paris 202 pp.

1995b« La politique du terrain ». Enquêtes. EHESS, Marseille, nº 1.

2003 « Les trois approches en anthropologie du développement » Revue Tiers Monde t. XLII, $n^{\circ} 168$ Paris

OTTINO (P.)

1972 Rangiroa. Parenté étendue, résidence et terres dans un atoll des Tuamotu. Cujas, Paris. $382 \mathrm{pp}$.

\section{PARTRIDGE (W.)}

1995 « Comments on 'including culture in evaluation research’ by Robert Klitgaard ». In Picciotto and Rist (eds.) Evaluation and Development. The World Bank, Washington 228-230

PERROT (J.-C.)

1992 Une histoire intellectuelle de l'économie politique. Editions de l’E.H.E.S.S., Paris. 508 pp.

\section{SAHLINS (M.)}

1980 Au cœur des sociétés. Raison pratique et raison culturelle. (trad. française de Culture and Practical Reason, Chicago, the University of Chicago Press, 1976.) 303 PP.

SEN (A.)

1988 « The Concept of Development » in H. Chenery and T.N. Srinivasan (eds.), Handbook of Development Economics Volume I, Elsevier Science Publishers B.V.. 
STIGLITZ (J.)

2002 La Grande Désillusion. Paris ; Fayard (traduction française de Globalization an dits Discontents, W.-W Norton) 324 pp.

TERRAY (E.)

1988 Lettres à la fugitive. Odile Jacob, Paris 220 pp.

VEYNE (P.)

1971 Comment on écrit l’histoire. « Points», Seuil, Paris. 242 pp. 